\title{
Comparison of the structural and orientational glass-transition dynamics in ethanol
}

\author{
M. A. Miller \\ Department of Physics and Astronomy and Center for Fundamental Materials Research, Michigan State University, \\ East Lansing, Michigan 48824 \\ M. Jimenez-Ruiz \\ Department of Physics and Astronomy and Center for Fundamental Materials Research, Michigan State University, \\ East Lansing, Michigan 48824 \\ and Instituto de Estructura de la Materia, Consejo Superior de Investigaciones Cientifica, Serrano 123, E-28006 Madrid, Spain \\ F. J. Bermejo \\ Instituto de Estructura de la Materia, Consejo Superior de Investigaciones Cientifica, Serrano 123, E-28006 Madrid, Spain \\ Norman O. Birge \\ Department of Physics and Astronomy and Center for Fundamental Materials Research, Michigan State University, East Lansing, \\ Michigan 48824 \\ (Received 1 December 1997)
}

\begin{abstract}
The dynamics of the supercooled-liquid and rotator-phase crystal of the same material, ethanol, are compared from measurements of the complex dielectric susceptibility. The relaxation times for the two phases are very close and appear to cross at $96 \mathrm{~K}$, the temperature corresponding both to the structural glass transition of the supercooled liquid and to the orientational glass transition of the rotator phase. Above $96 \mathrm{~K}$, the relaxation time of the supercooled liquid decreases faster than that of the rotator phase, presumably due to the liberation of additional translational degrees of freedom in the former. The dielectric results, coupled with recent specificheat measurements of the two phases, suggest that the rotational degrees of freedom provide the dominant contribution to structural relaxation near the glass transition, while flow processes provide a smaller contribution. [S0163-1829(98)00726-7]
\end{abstract}

The dynamics of glass-forming liquids has been extensively studied during recent years from both the theoretical and experimental points of view. ${ }^{1}$ The dominant dynamical process governing the behavior of a glass-forming material is referred to as the $\alpha$ relaxation, and is identified as a structural relaxation. From an experimental point of view, the $\alpha$ relaxation displays some universal features: a departure from Debye-like behavior and a rapid increase in the relaxation time with decreasing temperature. The relaxation times $\tau(T)$ have non-Arrhenius temperature dependence and can typically be parametrized by means of the empirical VogelTamman-Fulcher law. ${ }^{2}$ Recent studies ${ }^{3}$ have identified such universalities in the glassy behavior of a class of materials known as orientationally disordered crystals (ODC's), whose molecular centers of mass possess long-range periodicity, yet retain orientational degrees of freedom. ${ }^{4}$ In comparison to true structural glasses, which are disordered both translationally and rotationally, the translational order of ODC's represents a significant reduction in the total number of degrees of freedom available to the material. This reduction of structural complexity should facilitate theoretical modeling, and thus ODC's are of great interest as model glass-forming systems. The remarkable similarity in behavior of some ODC's and supercooled liquids near the glass transition suggests that a direct way of experimentally addressing the most relevant phenomena of so-called "glassy behavior"' is to study a single polymorphic system that displays glassy behavior in two structurally dissimilar phases.
It has been known for two decades ${ }^{5}$ that ethanol $\left(\mathrm{C}_{2} \mathrm{H}_{5} \mathrm{OH}\right)$ exhibits interesting phase polymorphism, and appears to be quite unique in that it can be made to assume a variety of phases simply by varying the temperature in a controlled manner. The fully disordered liquid phase transforms into a structural glass (SG) upon rapid cooling. The orientationally disordered crystalline phase transforms from a rotator phase (RP) to an orientational glass (OG) upon cooling at a moderate rate. Finally, the stable, orientationally ordered crystal is produced either by very slow cooling of the liquid or by annealing the RP or supercooled liquid. Recent X-ray, neutron-diffraction, and Raman-spectroscopic ${ }^{6,7}$ studies have confirmed the existence of an orientational glasslike transition in the RP crystal at nearly the same temperature $\left(T_{g}=97 \mathrm{~K}\right)$ observed for the supercooled liquid-tostructural glass transition. The close similarity of highfrequency dynamical behavior in the orientational-glass and structural-glass phases has been established by neutron timeof-flight spectroscopy and low-temperature specific-heat measurements. $^{8}$ These properties, combined with the fact that the RP crystal can be readily prepared from the structural glass, suggest that ethanol provides a unique opportunity to investigate and compare the relaxation dynamics of both phases near their respective glass transitions. Such a comparison may help to clarify the role of translational and rotational degrees of freedom in the dynamical response of a material approaching the glass transition.

We have measured the complex dielectric susceptibility ( $\left.\varepsilon=\varepsilon^{\prime}+i \varepsilon^{\prime \prime}\right)$ of the supercooled-liquid and rotator-crystal 
phases of ethanol near their respective glass transitions. We find that the dielectric relaxation in both the supercooledliquid (SCL) and RP crystal is nonexponential, and that parameters characterizing the relaxation (e.g., degree of nonDebyeness and average relaxation time) can be approximated using a Cole-Davison ${ }^{9}$ fitting function. For a given temperature, comparison of the dielectric response in each phase indicates a steeper temperature dependence of the average relaxation time in the SCL relative to that of the RP crystal, and the relaxation times of both phases are nearly equal at $T=96 \mathrm{~K}$.

Preparing ethanol in the supercooled-liquid and rotatorcrystal phase follows an established protocol. ${ }^{6}$ Rapid cooling of the liquid at a rate greater than $6 \mathrm{~K} / \mathrm{min}$ bypasses crystallization at $T_{M}=159 \mathrm{~K},{ }^{10}$ and allows entry into the supercooled-liquid regime. Continuing to cool at the prescribed rate leads to the structural-glass transition at $T_{g}$ $=97 \mathrm{~K}$. The RP crystal (body-centered-cubic, lattice constant $a=5.37 \AA$ ) is then formed by warming and annealing in the temperature range $102-110 \mathrm{~K}$. The exact temperature and annealing time required for conversion to the RP crystal depends on the warming rate, as well as the initial cooling rate into the glassy state. ${ }^{5}$

Measurement of the frequency- and temperaturedependent complex dielectric susceptibility was carried out using a sealed coaxial capacitor identical in design to that used in our previous work. ${ }^{3}$ Two different measurement schemes were employed depending on the frequency range under consideration. In the range $1 \mathrm{mHz}$ to $10 \mathrm{kHz}$, the inphase and out-of-phase currents through the capacitor were measured using a Stanford SR850 Dual Phase Lock-in Amplifier in conjunction with a Stanford SR570 Current-toVoltage Amplifier. For frequencies of $1 \mathrm{kHz}$ to $10 \mathrm{MHz}$, a Hewlett-Packard 4192A Impedance Analyzer was used in a standard four-terminal pair configuration to measure the capacitance and conductance of the sample. Data obtained from the two methods are in excellent agreement where they overlap.

Sample material consisted of 200-proof, dehydrated ethyl alcohol (ethanol) obtained from Quantum Chemical Company of Tuscola, Illinois. To avoid contamination with water vapor, filling of the capacitor was carried out at room temperature in a dry-nitrogen atmosphere. The sample cell was then frozen, evacuated of remaining nitrogen gas, and sealed. The sample cell was then mounted inside a nested cryocan arrangement described elsewhere. ${ }^{3}$ Finally, the entire cryocan assembly was immersed in a double-walled glass Dewar. This design provided control of the sample temperature to within $0.05 \mathrm{~K}$ over the range $4-300 \mathrm{~K}$, and minimized the occurrence of temperature gradients across the sample.

Figures 1(a) and 1(b) show our data for the real $\left(\varepsilon^{\prime}\right)$ and imaginary $\left(\varepsilon^{\prime \prime}\right)$ parts of the dielectric susceptibility for the supercooled liquid as a function of frequency for temperatures of 96, 98, and $100 \mathrm{~K}$. Figures 2(a) and 2(b) show the corresponding data taken in the RP crystal at temperatures of 96, 98, 100, 102, 104, 106, 108, and $110 \mathrm{~K}$. Although the glass-transition temperature is usually quoted as $T_{g}=97 \mathrm{~K}$, we emphasize that all our data are taken in the metastable equilibrium SCL and RP, respectively. The data at $96 \mathrm{~K}$, in particular, were observed not to change over more than $1 \mathrm{~h}$, indicating that no structural relaxation was taking place. Ob-

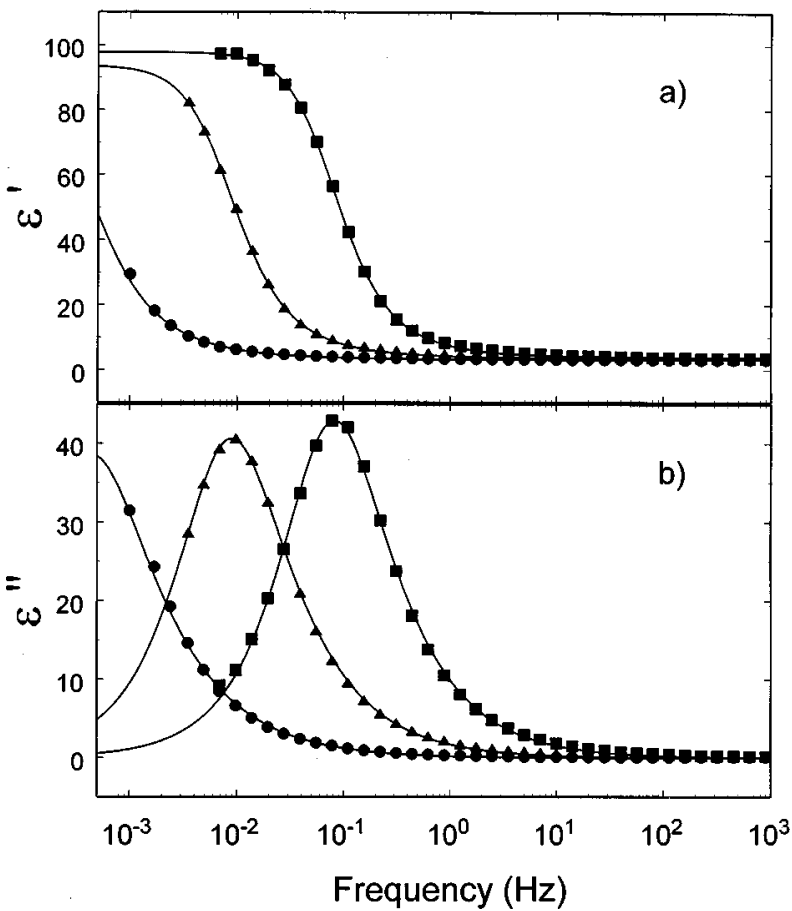

FIG. 1. (a) $\varepsilon^{\prime}$ and (b) $\varepsilon^{\prime \prime}$ vs frequency for temperatures of 96 $\boldsymbol{O}), 98(\mathbf{\Delta})$, and $100 \mathrm{~K}(\boldsymbol{\square})$ in the supercooled-liquid phase of ethanol. Solid lines are fits to the Cole-Davidson function.

taining data over a broad temperature range in the SCL phase was hindered by the fact that time-dependent transitions to the RP crystal occur above $102 \mathrm{~K}$. Thus, in order to broaden the range of liquid-phase data, additional measurements were

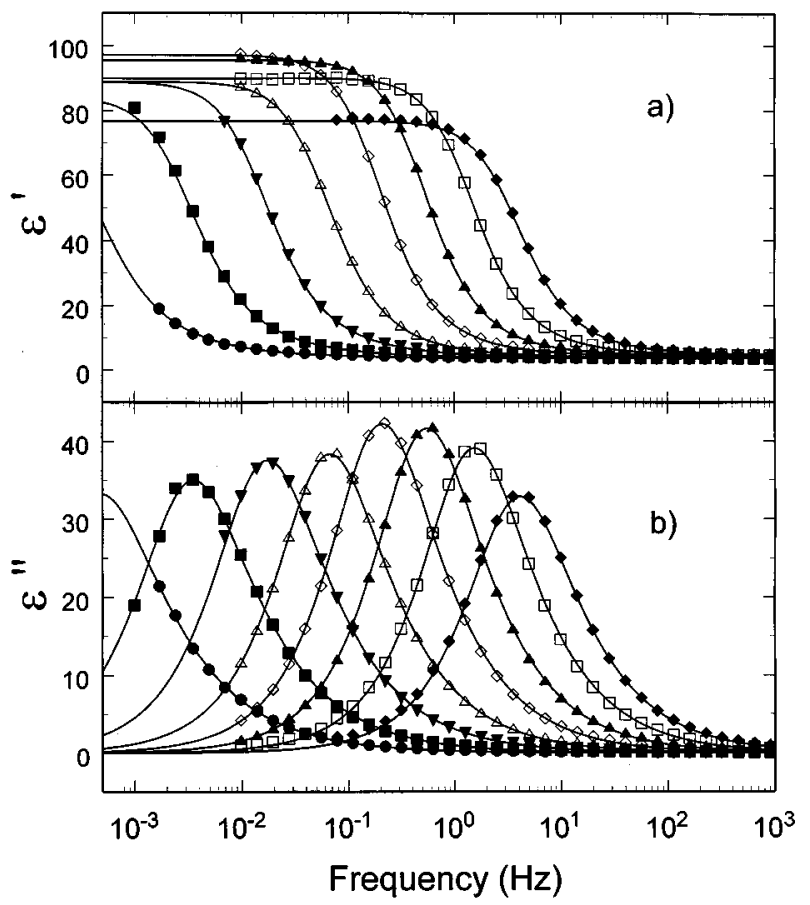

FIG. 2. (a) $\varepsilon^{\prime}$ and (b) $\varepsilon^{\prime \prime}$ vs frequency for temperatures of 96 $(\bullet), 98(\mathbf{\square}), 100(\boldsymbol{\nabla}), 102(\triangle), 104(\diamond), 106(\mathbf{\Delta}), 108(\square)$, and $110 \mathrm{~K}(\checkmark)$ in the rotator-crystal phase of ethanol. Solid lines are fits to the Cole-Davidson function. 


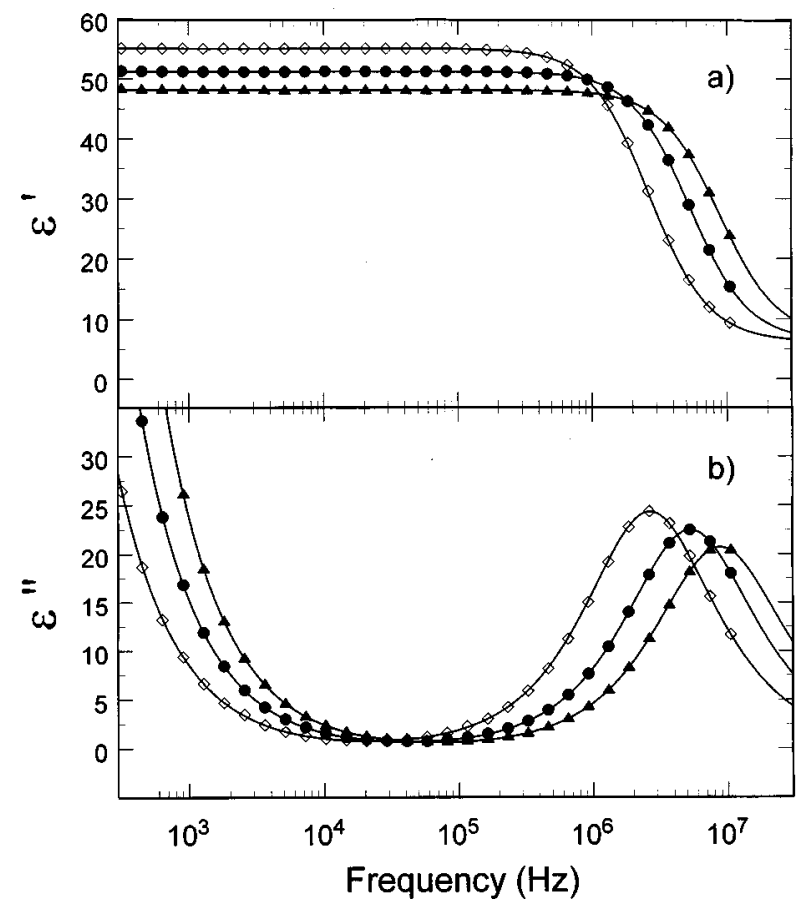

FIG. 3. (a) $\varepsilon^{\prime}$ and (b) $\varepsilon^{\prime \prime}$ vs frequency for temperatures of 164 $(\diamond), 173(\bullet)$, and $181 \mathrm{~K}(\mathbf{\Delta})$ in the liquid phase of ethanol. Solid lines are fits to the Cole-Davidson function plus a term of the form $\sigma_{\mathrm{dc}} / \omega$ in $\varepsilon^{\prime \prime}$ due to de conductivity.

made in the temperature range 164-181 K. Figures 3(a) and 3(b) show the high-temperature liquid-phase data, where the contribution of dc conductivity to the response at low frequencies is readily apparent.

The solid lines passing through the data in Figs. 1-3 are fits to the Cole-Davidson fitting form ${ }^{9}$ given by

$$
\varepsilon(\omega)=\varepsilon_{\infty}+\left(\varepsilon_{0}-\varepsilon_{\infty}\right) /\left(1-i \omega / \omega_{p}^{\mathrm{CD}}\right)^{\alpha},
$$

where $\omega=2 \pi f$. It is well known that fitting relaxation data with empirical forms such as the Cole-Davidson or Kohlraush-Williams-Watts function ${ }^{11}$ does not accurately describe the relaxation at all frequencies. In particular, at frequencies higher than two decades or so above the peak, the data typically obey a shallow power-law dependence extending to very high frequency. ${ }^{12,13}$ Nevertheless, the ColeDavidson form can be used in the main peak region to provide estimates of parameters characterizing the dynamical response such as the mean relaxation time $\left(\tau \approx 1 / \omega_{p}^{\mathrm{CD}}\right)$, and $\alpha$, the degree of departure from a Debye-governed relaxation process $(\alpha=1)$.

Figure 4 shows the temperature dependence of the width parameter $\alpha$ for both the SCL and RP crystal. Both phases display a characteristic feature common to many glassforming materials, namely, a decrease of $\alpha$ with temperature indicating deviation from idealized Debye behavior. ${ }^{1}$ Figures 1 and 2 show that the rotational contribution to the static susceptibility, $\varepsilon_{0}-\varepsilon_{\infty}$, is similar in the two phases, indicating that the molecules undergo complete rotation in the SCL as they do in the RP crystal. A surprising feature of the data in Figs. 1 and 2 is the decrease in $\varepsilon_{0}-\varepsilon_{\infty}$ at low temperature. This cannot be due to partial crystallization of the sample during the measurement because the data in Fig. 1 were

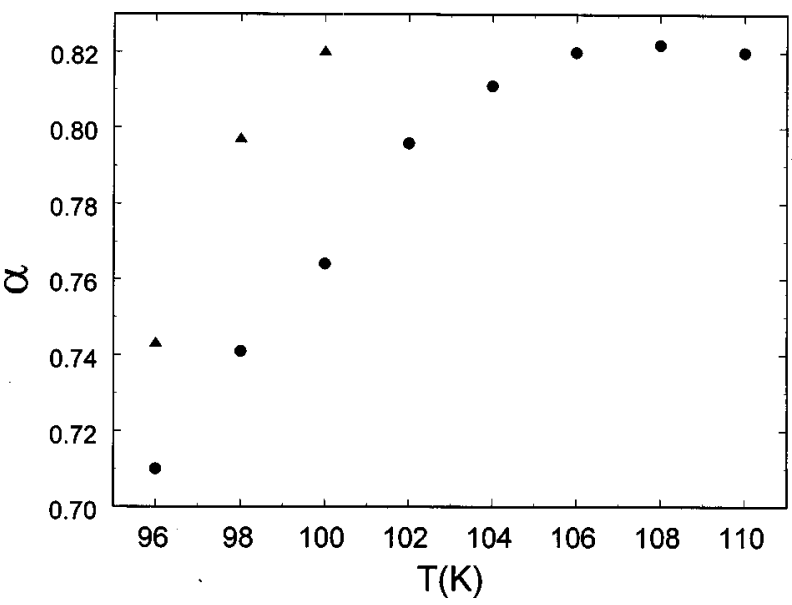

FIG. 4. Plot of the Cole-Davidson exponent $\alpha$ vs temperature for both the supercooled-liquid $(\mathbf{\Delta})$ and rotator-crystal $(\mathbf{O})$ phases. Both phases exhibit increasingly non-Debye behavior as the glass transition is approached, a common characteristic of many glassforming materials.

taken on warming from the SG, and the data in Fig. 2 were verified to be reproducible on warming from the OG.

Figure 5 shows the temperature dependence of the mean relaxation time in both the RP crystal and SCL phases. The solid lines are fits to the Vogel-Tamman-Fulcher (VTF) equation ${ }^{2}$ given by

$$
\tau=\tau_{0} \exp \left[A /\left(T-T_{0}\right)\right] .
$$

Here, $\tau_{0}$ is an attempt time and $T_{0}$ is the Vogel temperature at which the relaxation time diverges. Results of the VTF

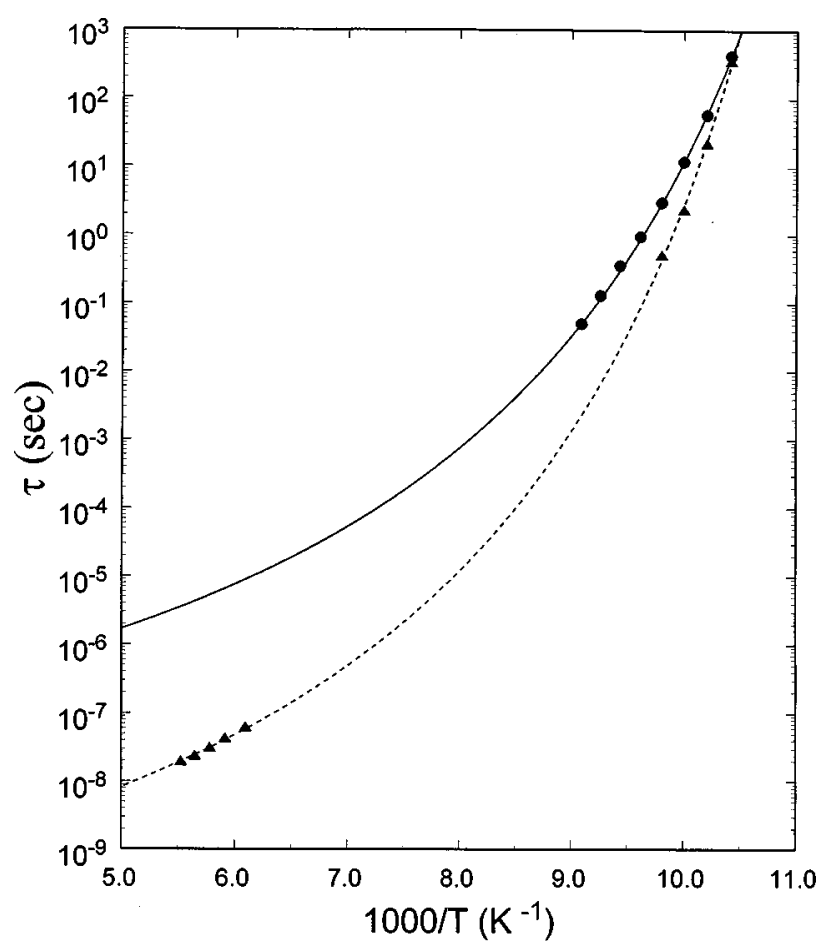

FIG. 5. The mean relaxation time $\tau$ vs inverse temperature for the liquid and supercooled-liquid $(\mathbf{\Delta})$ and rotator-crystal $(\mathbf{O})$ phases of ethanol. The relaxation times cross at $96 \mathrm{~K}$, near the glasstransition temperature. 
TABLE I. VTF fit parameters of the mean relaxation time in the RP crystal and SCL phases of ethanol.

\begin{tabular}{lccc}
\hline \hline Parameter & $A$ & $T_{0}$ & $\tau_{0}$ \\
\hline SCL & 596 & 75.6 & $6.8 \times 10^{-11}$ \\
RP crystal & 529 & 73.5 & $2.6 \times 10^{-8}$ \\
\hline
\end{tabular}

fitting for each phase are listed in Table I. The mean relaxation times in the two phases are nearly equal at $T=96 \mathrm{~K}$, and display a temperature-dependent shift toward a longer average relaxation time in the RP crystal phase relative to the SCL with increasing temperature. This manifests itself experimentally as a pronounced shift of the RP crystal relaxation peak toward lower frequency relative to the relaxation peak in the SCL at the same temperature. This behavior can be explained by considering the degrees of freedom available to the material in passing through the glass transition in each phase. The $\mathrm{OG} \rightarrow \mathrm{RP}$ transition involves the liberation of purely rotational degrees of freedom, while the $\mathrm{SG} \rightarrow \mathrm{SCL}$ transition involves liberation of both rotational and translational degrees of freedom. As the temperature increases above $96 \mathrm{~K}$, the additional translational freedom present in the SCL provides the constituent molecules with a less restrictive local environment, leading to increasingly shorter relaxation times in the SCL relative to the RP crystal for a given temperature. Recent specific-heat measurements ${ }^{5,14}$ performed on both the SCL and RP crystal near their respective glass transitions support this argument: the $\mathrm{OG} \rightarrow \mathrm{RP}$ crystal transition involves an increase of about $22 \mathrm{~J} \mathrm{~mol}^{-1} \mathrm{~K}^{-1}$ and the $\mathrm{SG} \rightarrow \mathrm{SCL}$ transition involves an in- crease in the specific heat of approximately $31 \mathrm{~J} \mathrm{~mol}^{-1} \mathrm{~K}^{-1}$. Presumably the extra $9 \mathrm{~J} \mathrm{~mol}^{-1} \mathrm{~K}^{-1}$ in the SCL specific heat not present in the RP crystal corresponds to translational degrees of freedom. These results suggest that the rotational degrees of freedom are the dominant contributor to structural relaxation processes near the glass transition in both the RP crystal and SCL, and that flow processes such as mass diffusion associated with translational degrees of freedom in the SCL contribute to a lesser extent.

In conclusion, we have used dielectric spectroscopy to compare the dynamical response of the SCL and RP crystal phases of ethanol. We find that the orientational-glass transition in RP crystal exhibits all the characteristics observed in the structural-glass transition: an increasingly non-Debye relaxation and faster-than-Arrhenius behavior in the relaxation time as the glass transition is approached. Comparison of the relaxation time behavior above $96 \mathrm{~K}$ displays the effect of the additional translational freedom available to the liquid resulting in faster molecular reorientation in the SCL relative to the RP crystal for a given temperature. The dielectric data indicate that rotational motion is the dominant dynamical process governing the glass transition in both SCL and RP crystal ethanol. Thus, the construction of a minimal model utilizing restricted degrees of freedom now appears relevant to the behavior of real materials. A model such as that explored by Renner, Lowen, and Barrat ${ }^{15}$ involving hard needles on an fcc lattice, should be simple enough to allow a detailed numerical and analytical treatment.

We are grateful to S. R. Nagel and D. L. Price for their role in establishing this collaboration. F.J.B. and M.J.R. acknowledge support from DGICYT Grant (Spain) No. PB950075-C03-01.
${ }^{1}$ M. D. Ediger, C. A. Angell, and S. R. Nagel, J. Phys. Chem. 100, 13200 (1996).

${ }^{2}$ H. Vogel, Z. Phys. 22, 645 (1921); G. S. Fulcher, J. Am. Ceram. Soc. 8, 339 (1925); G. Tamman and W. Hesse, Z. Anorg. Allg. Chem. 156, 245 (1926).

${ }^{3}$ D. L. Leslie-Pelecky and N. O. Birge, Phys. Rev. Lett. 72, 1232 (1994); Phys. Rev. B 50, 13250 (1994).

${ }^{4} \mathrm{~J}$. N. Sherwoord, The Plastically Crystalline State (Orientationally-Disordered Crystals) (Wiley, New York, 1979).

${ }^{5}$ O. Haida, H. Suga, and S. Seki, J. Chem. Thermodyn. 9, 1133 (1977).

${ }^{6}$ A. Srinivasan, F. J. Bermejo, A. de Andres, J. Dawidowski, J. Zuniga, and A. Criado, Phys. Rev. B 53, 8172 (1996).

${ }^{7}$ R. Fayos, F. J. Bermejo, J. Dawidowski, H. E. Fischer, and M. A. Gonzalez, Phys. Rev. Lett. 77, 3823 (1996).

${ }^{8}$ M. A. Ramos, S. Vieira, F. J. Bermejo, J. Dawidowski, H. E. Fischer, H. Schober, M. A. Gonzalez, C. K. Loong, and D. L.
Price, Phys. Rev. Lett. 78, 82 (1997).

${ }^{9}$ D. W. Davidson and R. H. Cole, J. Chem. Phys. 18, 1417 (1951).

${ }^{10} \mathrm{~A}$ typographical error in Ref. 5 giving $T_{m}$ as $169 \mathrm{~K}$ has been propagated in some papers, e.g., Refs. 6 and 7.

${ }^{11}$ G. Williams and D. C. Watts, Trans. Faraday Soc. 66, 80 (1970).

${ }^{12}$ P. K. Dixon, L. Wu, S. R. Nagel, B. D. Williams, and J. P. Carini, Phys. Rev. Lett. 65, 1108 (1990).

${ }^{13}$ R. Brand, P. Lunkenheimer, and A. Loidl, Phys. Rev. B 56, 5713 (1997).

${ }^{14}$ F. J. Bermejo, A. Criado, R. Fayos, R. Fernandez-Perea, H. E. Fischer, E. Suard, A. Guelylah, and J. Zuniga, Phys. Rev. B 56, 11536 (1997); F. J. Bermejo, H. E. Fischer, M. A. Ramos, A. de Andres, J. Davidowski, and R. Fayos, in Complex Behaviour of Glassy Systems, edited by M. Rubi et al., Springer Lecture Notes in Physics Vol. 492 (Springer-Verlag, Berlin, 1997), p. 44.

${ }^{15}$ C. Renner, H. Lowen, and J. L. Barrat, Phys. Rev. E 52, 5091 (1995). 